\title{
FITOPLANCTON DEL REFUGIO DE FAUNA SILVESTRE ISLA DE AVES: EL TERRITORIO VENEZOLANO MÁS SEPTENTRIONAL
}

\section{Phytoplankton of the Wildlife Refuge Isla de Aves: the northern most Venezuelan territory}

\section{Carlos Pereira}

Dirección Ejecutiva de Ambiente, Petróleos de

Venezuela, S.A., Caracas. Venezuela

\section{Rubén Quiñones}

Servicio de Hidrografía y Navegación, Armada

Bolivariana de Venezuela, Caracas. Venezuela

\author{
Vanessa Hernández \\ Dirección Ejecutiva de Ambiente, Petróleos de \\ Venezuela, S.A., Caracas. Venezuela
}

Recibido: Agosto 20, 2018 Aprobado: Septiembre 24, 2018

Cómo citar: Pereira, C., Quiñones, R., \& Hernández, V. (2018). Fitoplancton del Refugio de Fauna Silvestre Isla de Aves: el territorio venezolano más septentrional. Ciencia, Ambiente Y Clima, 1(1), 45-59. https://doi.org/10.22206/ cac.2018.v1i1.pp45-59

\section{Resumen}

Como parte de una estrategia de conservación del Refugio de Fauna Silvestre Isla de Aves, el cual representa una zona geoestratégica para la República Bolivariana de Venezuela, las instituciones de investigación del país realizan campańas anuales para conocer la biodiversidad de la isla. Uno de los componentes biológicos más importantes es el fitoplancton, ya que, a pesar de que sustenta las redes tróficas pelágicas marinas y es parte esencial del ciclo de los nutrientes, no ha sido estudiado en esta zona, por lo que se presenta en este documento, un inventario del fitoplancton marino. Para ello, se realizaron arrastres con una red cónica de fitoplancton en julio de 2011 y 2012. En laboratorio, se estimó la abundancia por el método de Utermölh, con un microscopio invertido y claves taxonómicas. La comunidad estuvo representada por 191 especies distribuidas en 121 diatomeas, 58 dinoflagelados, 6 cianobacterias, 3 euglenofitas y 3 silicoflagelados. Finalmente, destacamos que la zona marino costera de la isla se caracteriza por una comunidad fitoplanctónica diversa, con abundancias características de aguas oligotróficas de ecosistemas coralinos y procesos de mezcla de la columna de agua.

Palabras clave: Caribe venezolano, ecología marina, fitoplancton, Isla de Aves, microalgas.

\begin{abstract}
As part of a conservation strategy for the Wildlife Refuge "Isla de Aves", which represents a geostrategic zone for the Bolivarian Republic of Venezuela, several research institutions conduct studies every year to learn about the island's biodiversity. One of the most important biological components is phytoplankton, since although it sustains marine pelagic trophic networks and is an essential part of the nutrient cycles; it has not been studied in this area, so an inventory of marine phytoplankton was accomplished. Here, we carried out several trawls with a conical phytoplankton net in July 2011 and July 2012. In the laboratory, abundance was estimated by the Utermölh method, with an inverted microscope and specialized bibliography. The community was represented by 191 species distributed in 121 diatoms, 58 dinoflagellates, 6 cyanobacteria, 3 eugleophytes and 3 silicoflagellates. The coastal marine zone of the island is characterized by a diverse phytoplankton community, with characteristic abundances of oligotrophic waters of coral ecosystems and processes of mixing the water column.
\end{abstract}

Keywords: Venezuelan Caribbean, marine ecology, phytoplankton, Island of birds, microalgae. 


\section{Introducción}

El Refugio de Fauna Silvestre Isla de Aves es considerado un bastión geoestratégico de gran importancia para la República Bolivariana de Venezuela, ya que representa la porción de tierra sobre el nivel de mar más septentrional del país, de la cual se derivan más de $130.000 \mathrm{Km}^{2}$ de mar territorial, plataforma continental y zona económica exclusiva. Está ubicada en el Mar Caribe (15 40' 23,7" N 63 36' 59,9 " W), a $435 \mathrm{Km}$ al norte de la Blanquilla, 650 $\mathrm{Km}$ al noreste del Puerto de La Guaira, $520 \mathrm{Km}$ al Norte de la Isla de Margarita, $200 \mathrm{Km}$ al oeste de Dominica y $350 \mathrm{Km}$ al sureste de Puerto Rico (Hubschmann et al., 1988).

Isla de Aves pertenece a una formación geológica consolidada que se denomina Promontorio de Aves, el cual, cubre un área extensa que se proyecta al norte del territorio insular venezolano. Esta cadena submarina está asociada a la subducción, sobrecorrimiento y desplazamiento de la Placa del Caribe o Caribia, la cual, ha sufrido una evolución histórica a partir de un levantamiento inicial y rápido de la tierra, producido por actividad volcánica, seguido de erosión gradual y subsidencia. A pesar de esto, diversos investigadores afirman que el crecimiento de arrecifes coralinos supera a la subsidencia geológica de la zona, por lo menos en un orden de magnitud (Gruber, 2003).

Debido a su alta diversidad biológica, incluyendo la de aves migratorias, y por ser el sitio de anidación de la tortuga verde (Chelonia mydas) más importante del país, y el segundo en el mar Caribe, luego del Parque Nacional de Tortuguero en Costa Rica (Guada y Solé, 2000, p. 22); en 1972 fue decretada Refugio de Fauna Silvestre. Sin embargo, se han realizado pocos estudios sobre sus comunidades marinas, destacándose el de Yranzo, Villamizar, Romero y Boadas (2014), en el que se identificaron 36 especies de corales pétreos y cuatro de hidrocorales, siendo Porites astreoides, Pseudodiploria strigosa y Siderastrea siderea las especies más abundantes, con una cobertura promedio de coral vivo estimada de $22,30 \%$.

Una de las comunidades que sustenta la alta biodiversidad de este ecosistema marino es el fitoplancton, del cual no se han publicado estudios. Las comunidades fitoplanctónicas incluyen a las microalgas suspendidas en la columna de agua que están a la merced de la dinámica costera. Este grupo de organismos fotosintetizadores constituye la base de la trama alimentaria en la mayor parte de las comunidades marinas, ya que además de utilizar los nutrientes elementales del agua y transformarlos en materia orgánica (Yilmaz, Yardimci, Elhag \& Dumitrache, 2018), es responsable en gran proporción de la productividad primaria oceánica (Arrigo et al., 1999; Luz y Barkan, 2000). Asimismo, el fitoplancton es capaz de responder rápidamente ante las fluctuaciones hidrográficas, explicando la mayoría de los procesos oceánicos y es considerado, el principal regulador de oxígeno y dióxido de carbono de la atmósfera (Troccoli, 2001). Son estas las razones por lo que se planteó hacer un inventario de las especies de microalgas que constituyen la comunidad fitoplanctónica del refugio de fauna silvestre Isla de Aves.

\section{Materiales y Métodos}

\section{1 Área de estudio}

Isla de Aves es una Dependencia Federal Venezolana de forma alargada en sentido norte-sur, con su parte central más angosta. Posee $600 \mathrm{~m}$ de longitud en sentido norte-sur y $130 \mathrm{~m}$ en sentido este-oeste con una superficie de $42.000 \mathrm{~m}^{2}$ (ver figura 1). Es un área afectada por el paso de tormentas y huracanes, $y$ ha sido considerada de bajo impacto antropogénico, debido a que no tiene presión de pesca y turismo, con un desarrollo de actividades humanas muy bajo, ya que la población residente en la isla es de 15 personas aproximadamente (Yranzo et al., 2014). 


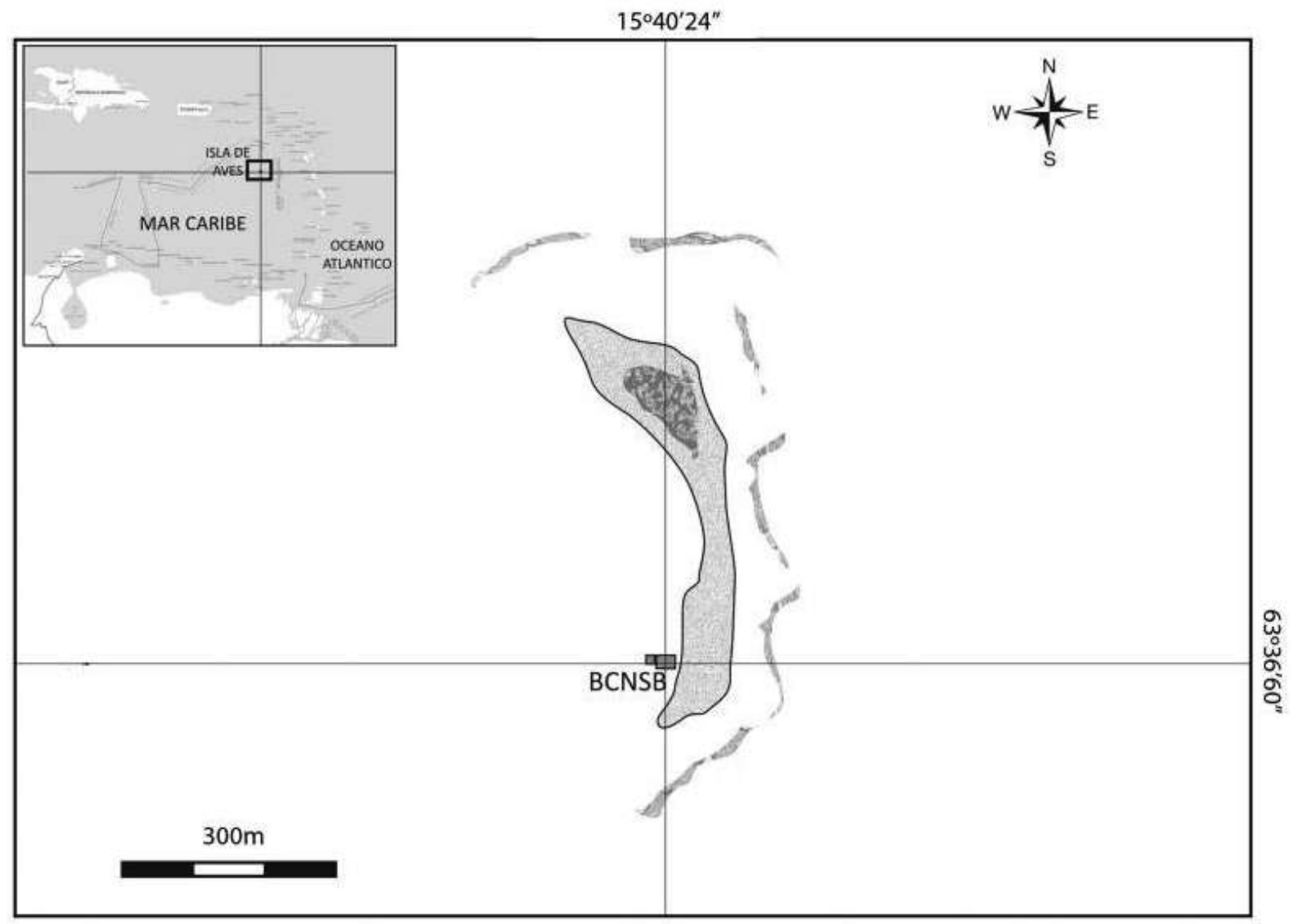

Figura $\mathbf{N}^{\circ}$ 1. Mapa del refugio de fauna silvestre de Isla de Aves Fuente: Yranzo et al., 2014, p. 116.

\subsection{Obtención de datos}

Para la captura del fitoplancton, se realizaron 16 arrastres entre 200 y $300 \mathrm{~m}$ alrededor de la isla, con una red cónica de plancton con diámetro de malla de $60 \mu \mathrm{m}$, en julio de 2011 y en julio de 2012. Cada arrastre tuvo una duración de 10 minutos aproximadamente y mediante el empleo de una embarcación inflable con motor fuera de borda, a una velocidad promedio de $5 \mathrm{~km} / \mathrm{h}$. La red se arrastró en la zona superficial de la columna de agua en horas de la mañana. Las muestras fueron recolectadas en envases de $500 \mathrm{ml}$ y fijadas in situ con una solución de lugol y formalina neutralizada al 5\% v/v.

En el laboratorio, se estimó la abundancia de las microalgas por el método de Utermölh (1958), mediante el uso de cámaras de sedimentación y un microscopio invertido marca Leica. La identificación taxonómica de las especies de microalgas se realizó utilizando los trabajos de Cupp (1943), Peragallo (1965), Ferguson (1968), Saunders y Glenn (1969), Sournia (1986), Balech (1988), Round, Crawford y Mann (1990), ChretiennotDinet Sournia, Ricard y Billard (1993), Tomas (1996), Yamaji (1996), Berárd-Therriault, Poulin y Bossé (1999), Soler, Pérez y Aguilar (2003), AlYamani y Saburova (2011), Siqueiros-Beltrones, Valenzuela, Hernández, Argumedo \& López, (2004), Siqueiros-Beltrones y Hernández (2006), entre otros. La revisión y actualización de los nombres científicos se realizó en la página web de Algaebase.org (Guiry \& Guiry, 2018).

Con los resultados obtenidos, se elaboró una matriz de las especies del fitoplancton, en la cual se 
incluye su abundancia y riqueza. Posteriormente, se elaboró una matriz de similaridad, usando la distancia de Bray Curtis para cuantificar la contribución de cada especie a la disimilaridad temporal de la estructura comunitaria del fitoplancton; $y$ un ANOSIM, para estimar si existen diferencias significativas entre las muestras. Los análisis estadísticos se realizaron con el paquete PAST 3.2 (Hammer, Harper \& Ryan, 2001).

\section{Resultados}

En las muestras analizadas, se identificaron 191 especies distribuidas en 121 diatomeas (63\% de la comunidad), 58 dinoflagelados (30\%), 6 cianobacterias (3\%), 3 euglenofitas (2\%) y 3 silicoflagelados (2\%). El grupo de las diatomeas se distribuyó en 3 clases, 29 órdenes y 45 familias, mientras que los dinoflagelados en 2 clases, 8 órdenes y 15 familias; las cianobacterias en 1 clase, 3 órdenes y 5 familia; las euglenofitas en 2 clase, 2 órdenes y 3 familias; y los silicoflagelados en 1 clase, 2 órdenes y 3 familias, tal como se indica en la lista de especies. Las especies más frecuentes fueron las diatomeas: Asterionellopsis glacialis, Asteroplanus karianus, Chaetoceros dydimus, Cocconeis ornata, Coscinodiscus perforatus, Haslea wawrikae, Isthmia enervis, Lauderia annulata, Leptocylindrus danicus, Leptocylindrus minimus, Lyrella lyra, Nitzschia sp., Pseudoguinardia recta, Rhizosolenia styliformis, Skeletonema costatum, Thalassiosira nordenskioldii; la cianobacteria: Trichodesmium thiebauthii; los dinoflagelados: Gymnodinium cinctum, Ornithocercus magnificus, Ostreopsis ovata, Ostreopsis siamenses, Tripos spp., y las euglenofitas: Phacus sp. y Eutreptiella gymnastica.

En cuanto a la abundancia, durante los muestreos realizados en el ańo 2011 se obtiene una media de 1999 células/litro, de las cuales las diatomeas contribuyen con el $68 \%$, siendo las especies más importantes: Leptocylindrus minimus, Chaetoceros dydimus, Leptocylindrus danicus, Asteroplanus karianus, Nitzschia sp.1, Coscinodiscus perforatus, Licmophora abreviatta, Prosbocia alata, Skeletonema costatum, Haslea wawrikae y Cylindrotheca closterium. El segundo grupo más importante es el de las cianobacterias con un $27 \%$ de la abundancia, siendo Trichodesmium thiebauthii la especie más importante. A pesar que los dinoflagelados sólo representan cerca del $4 \%$ de la abundancia, la especie que contribuye en mayor proporción a este porcentaje es Tripos azoricum. En el año 2012, la abundancia fue muy similar al año anterior, obteniéndose una media de 1,546 células/litro, en la que las diatomeas contribuyeron en un $62 \%$ con las especies Skeletonema costatum, Pseudoguinardia recta y Leptocylindrus danicus, seguidas por las cianobacterias con un 33\% y los dinoflagelados con $4 \%$, siendo las especies más importantes, la cianobacteria: Trichodesmium thiebauthii y los dinoflagelados: Ostreopsis ovata, Gymnodinium cinctum y Scrippsiella acuminata.

$\mathrm{Al}$ aplicar una prueba Anosim, para establecer las diferencias de la comunidad fitoplanctónica en los dos ańos muestreados, se obtuvo que no hay diferencias significativas $(\mathrm{R}=0,25 ; \mathrm{p}=2,1 \%)$. La prueba SIMPER indica una similaridad entre las muestras del año 2011 de $42,5 \%$ en la que Trichodesmium thiebauthii, Asteroplanus karianus, Coscinodiscus perforatus, Skeletonema costatum, Nitzschia sp. 1, Leptocylindrus danicus y Haslea wawrikae contribuyeron con más del $70 \%$ a la similaridad encontrada. Mientras que en 2012, la similaridad fue un poco menor $(25,6 \%)$, siendo las especies que contribuyeron en mayor proporción a esta similaridad Trichodesmium thiebauthii, Skeletonema costatum, Pseudoguinardia recta, Nostoc sp. y Leptocylindrus danicus. Al comparar ambos años, la similaridad fue del $21 \%$ con la contribución mayor de las especies mencionadas anteriormente.

A continuación, se presenta la lista de especies de microalgas identificadas en el refugio de fauna silvestre de Isla de Aves. 


\section{Diatomeas}

PHYLUM: BACILLARIOPHYTA Karsten 1928 CLASE: BACILLARIOPHYCEAE Haeckel 1878

ORDEN: Bacillariales Hendey 1937 emend. Round et al. 1990

FAMILIA: Bacillariaceae Ehrenberg 1831

Bacillaria paxillifera (O. F. Müller) T. Marsson 1901

Cylindrotheca closterium (Ehrenberg) Reimann \& J. C. Lewin 1964

Nitzschia sp. Hassall

Nitzschia acicularis (Kützing) W. Smith 1853

Nitzschia angularis W. Smith 1853

Nitzschia fasciculata Grunow en Van Heurck 1881

Nitzschia hyalina Gregory 1857

Nitzschia incurvata var. lorenziana Ross 1986

Nitzschia lanceolata Smith 1853

Nitzschia longissima (Brébisson) Ralfs en

Pritchard 1861

Pseudo-nitzschia pungens (Grunow ex Cleve) Hasle 1993

Pseudo-nitzschia seriata (Cleve) H. Peragallo en H. Peragallo \& M. Peragallo 1899

ORDEN: Cocconeidales Cox 2015

FAMILIA: Cocconeidaceae Kützing 1844

Cocconeis ornata W. Gregory 1857

Cocconeis scutellum Ehrenberg 1838

ORDEN: Cymbellales Mann en Round, Crawford \& Mann 1990

FAMILIA: Gomphonemataceae Kützing 1844 Gomphonema acuminatum Ehrenberg 1832

ORDEN: Eunotiales Silva en Lewin 1962

FAMILIA: Eunotiaceae Kützing 1844

Eunotia sp. Ehrenberg 1837

ORDEN: Fragiliarales Silva 1962 emend. Round 1990

FAMILIA: Fragilariaceae Greville 1833

Asteroplanus karianus (Grunow) C. Gardner \& R.M.Crawford en R.M. Crawford \& C. Gardner 1997
Fragilaria Lyngbye 1819

Podocystis adriatica (Kützing) Ralfs en Pritchard 1861

ORDEN: Licmophorales Round 1990

FAMILIA: Licmophoraceae Kützing 1844

Licmophora abbreviate C. Agardh 1831

Licmophora gracilis (Ehrenberg) Grunow 1867

Licmophora remulus (Grunow) Grunow 1867

Licmophora tenuis (Kützing) Grunow 1867

FAMILIA: Ulnariaceae E. J. Cox 2015

Tabularia fasciculate (Agardh) Williams \&

Round 1986

Ulnaria capitata (Ehrenberg) Compère 2001

ORDEN: Lyrellales D. G. Mann 1990

FAMILIA: Lyrellaceae D. G. Mann 1990

Lyrella lyra (Ehrenberg) Karajeva 1978

ORDEN: Mastogloiales Mann 1990

FAMILIA: Achnanthaceae Kützing 1844

Achnanthes longipes Agardh 1824

ORDEN: Naviculales Bessey 1907

FAMILIA: Amphipleuraceae Grunow 1862

Halamphora turgida (Gregory) Levkov 2009

FAMILIA: Diploneidaceae Mann 1990

Diploneis crabro (Ehrenberg) Ehrenberg 1854

FAMILIA: Naviculaceae Kützing 1844

Gyrosigma attenuatum (Kützing) Rabenhorst 1853

Haslea ostrearia (Gaillon) Simonsen 1974

Haslea wawrikae (Husedt) R. Simonsen 1974

Navicula sp. Bory 1822

Navicula arenicola Grunow 1882

Navicula palpebralis Brébisson ex Smith 1853

Pinnunavis yarrensis (Grunow) Okuno 1975

FAMILIA: Pinnulariaceae Mann 1990

Pinnularia Ehrenberg 1843

FAMILIA: Plagiotropidaceae Mann en Round, Crawford \& Mann 1990

Plagiotropis lepidoptera (Gregory) Kuntze 1898 
FAMILIA: Pleurosigmataceae Mereschowsky 1903 Pleurosigma formosum W. Smith 1852

ORDEN: Rhabdonematales Round \& Crawford 1990

FAMILIA: Grammatophoraceae Lobban \& Ashworth 2014

Grammatophora marina (Lyngbye) Kützing 1844

Grammatophora oceanica Ehrenberg 1840

FAMILIA: Rhabdonemataceae Round \& Crawford 1990

Rhabdonema Kützing 1844

ORDEN: Rhaphoneidales Round in Round et al., 1990

FAMILIA: Asterionellopsidaceae, Medlin 2016 Asterionellopsis glacialis (Castracane) Round in Round, Crawford \& Mann 1990

FAMILIA: Psammodiscaceae Round \& D. G. Mann 1980

Psammodiscus nitidus (W.Gregory) Round \& D.

G. Mann 1980

ORDEN: Rophalodiales Mann 1990

FAMILIA: Rhopalodiaceae (Karsten) Topachevs'kyj \& Oksiyuk 1960 Epithemia Kützing 1844

ORDEN: Striatellales Round1990

FAMILIA: Striatellaceae Kützing 1844 Striatella unipunctata (Lyngbye) Agardh 1832

ORDEN: Surirellales D. G. Mann 1990

FAMILIA: AuriculaceaeHendey 1964 Auricula sp. Castracane 1873

FAMILIA: Entomoneidaceae Reimer en Patrick \& Reimer 1975

Entomoneis paludosa (Smith) Reimer en Patrick \& Reimer 1975

FAMILIA: Surirellaceae Kützing 1844 Campylodiscus neofastuosus Ruck \& Nakov en Ruck et al. 2016
Iconella biseriata (Brébisson) Ruck \& Nakov en Ruck et al. 2016

Surirella librile (Ehrenberg) Ehrenberg 1845

ORDEN: Tabellariales Round 1990

FAMILIA: Tabellariaceae Kützing 1844

Asterionella formosa Hassall 1850

Meridion circulare (Greville) Agardh 1831

ORDEN: Thalassiophysales Mann 1990

FAMILIA: Catenulaceae Mereschkowsky 1902

Cocconeis costata Gregory 1855

Amphora crassa Gregory 1857

Amphora ocellata Donkin 1861

Amphora proteus Gregory 1857

ORDEN: Thalassionematales Round 1990

FAMILIA: Thalassionemataceae Round 1990

Thalassionema nitzschioides (Grunow)

Mereschkowsky 1902

CLASE: COSCINODISCOPHYCEAE (Round \& Crawford 1990)

ORDEN: Aulacoseirales Crawford en Round, Crawford \& Mann 1990

FAMILIA: Aulacoseiraceae Crawford 1990 Aulacoseira crenulata (Ehrenberg) Thwaites 1848

ORDEN: Coscinodiscales Round \& Crawford en Round, Crawford \&Mann 1990

FAMILIA: Coscinodiscaceae Kützing 1844

Coscinodiscus perforates Ehrenberg 1844

Coscinodiscus wailesii Gran \& Angst 1931

Palmerina hardmaniana Hasle 1996

FAMILIA: Hemidiscaceae Hendey ex Hasle in Hasle \& Syvertsen 1996

Pseudoguinardia recta Stosch 1986

ORDEN: Melosirales Crawford in Round, Crawford \& Mann 1990

FAMILIA: Melosiraceae Kützing 1844

Melosira nummuloides Agardh 1824

ORDEN: Rhizosoleniales P. C. Silva en R. A. Lewin 1962 
FAMILIA: Probosciaceae Nikolaev \& Harwood en Nikolaev et al. 2001

Proboscia alata (Brightwell) Sundström 1986

FAMILIA: Rhizosoleniaceae De Toni 1890

Dactyliosolen fragilissimus (Bergon) Hasle en Hasle \& Syvertsen 1996

Guinardia delicatula (Cleve) Hasle en Hasle \& Syvertsen 1997

Guinardia fláccida (Castracane) H.Peragallo 1892

Guinardia striata (Stolterfoth) Hasle en Hasle \& Syvertsen 1996

Pseudosolenia calcar-avis Schultze Sundström 1986

Rhizosolenia acuminata $\mathrm{H}$. Peragallo en $\mathrm{H}$. Peragallo \& M. Peragallo 1907

Rhizosolenia castracanei Peragallo 1888

Rhizosolenia imbricata Brightwell 1858

Rhizosolenia obtusa Hensen 1887

Rhizosolenia setigera Brightwell 1858

Rhizosolenia styliformis Brightwell 1858

ORDEN: Triceratiales Round \& Crawford 1990

FAMILIA: Triceratiaceae (Schütt) Lemmermann 1899

Triceratium balearicum Cleve \& Grunow en Cleve 1881

Triceratium dubium Brightwell 1859

Triceratium favus Ehrenberg 1839

Triceratium gibbosum Harvey \& Bailey 1854

CLASE: MEDIOPHYCEAE (Jousé \& ProshkinaLavrenko) Medlin \& Kaczmarska 2004

ORDEN: Biddulphiales Krieger 1954

FAMILIA: Bellerocheaceae Crawford 1990 Bellerochea malleus (Brightwell) Van Heurck 1885

FAMILIA: Biddulphiaceae Kützing 1844 Biddulphia sp. Gray 1821

Biddulphia alternans (Bailey) Van Heurck 1885

ORDEN: Chaetocerotales Round \& Crawford en Round et al. 1990
FAMILIA: Chaetocerotaceae Ralfs en Pritchard 1861

Chaetoceros affinis Lauder 1864

Chaetoceros atlanticus Cleve 1873

Chaetoceros bacteriastrius G. C. Wallich

Chaetoceros coarctatus Lauder 1864

Chaetoceros compressus Lauder 1864

Chaetoceros costatus Pavillard 1911

Chaetoceros curvisetus Cleve 1889

Chaetoceros danicus Cleve 1889

Chaetoceros decipiens Cleve 1873

Chaetoceros dydimus Ehrenberg 1845

Chaetoceros gracilis Pantocsek 1892

Chaetoceros holsaticus F. Schütt 1895

Chaetoceros lorenzianus Grunow 1863

Chaetoceros peruvianus Brightwell 1856

Chaetoceros sociales Lauder 1864

Chaetoceros teres Cleve en Aurivillius 1896

Chaetoceros pseudocurvisetus Mangin 1910

FAMILIA: Leptocylindraceae Lebour 1930

Leptocylindrus danicusCleve 1889

Leptocylindrus minimusGran 1915

ORDEN: Cymatosirales Round \& Crawford en Round et al., 1990

FAMILIA: Cymatosiraceae Hasle, Stosch \& Syvertsen 1983

Plagiogrammopsis vanheurckii (Grunow) Hasle, Stosch \& Syvertsen 1983

ORDEN: Eupodiscales Nikolaev \& Harwood en Witkowski \& Sieminska 2000

FAMILIA: Eupodiscaceae Ralfs en Pritchard 1861

Odontella aurita (Lyngbye) Agardh 1832

Odontella obtusa Kützing 1844

Trieres regia (M.Schultze) Ashworth \& Theriot en Ashworth et al., 2013

ORDEN: Hemiaulales Round \& Crawford 1990

FAMILIA: Hemiaulaceae Heiberg 1863

Cerataulina bicornis (Ehrenberg) Hasle

Cerataulina pelagica (Cleve) Hendey 1937

Hemiaulus hauckii Grunow ex Van Heurck 1882

Ciencia, Ambiente y Clima 2018; 1(1): 45-59 
Hemialus membranaceus Cleve 1873

Hemialus sinensis Greville 1865

FAMILIA: Isthmiaceae Schütt 1896

Isthmia enervis Ehrenberg 1838

ORDEN: Lithodesmiales Round \& Crawford 1990

FAMILIA: Lithodesmiaceae Round 1990

Ditylum brigthwellii (T.West) Grunow en Van Heurck 1885

Lithodesmium undulatum Ehrenberg 1839

ORDEN: Thalassiosirales Glezer \& Makarova 1986

FAMILIA: Lauderiaceae (Schütt) Lemmermann 1899

Lauderia annulata Cleve 1873

FAMILIA: Skeletonemataceae Lebour 1930

Detonula pumila (Castracane) Gran 1900

Skeletonema costatum (Greville) Cleve 1873

FAMILIA: Thalassiosiraceae Lebour 1930 Planktoniella sol (Wallich) Schütt 1892 Thalassiosira eccentrica (Ehrenberg) Cleve 1904 Thalassiosira nordenskioeldii Cleve 1873

ORDEN: Toxariales Round 1990

FAMILIA: Climacospheniaceae Round 1990 Climacosphenia elongata Bailey 1854

\section{DINOFLAGELADOS}

PHYLUM: MIOZOA Cavalier-Smith 1987

CLASE: DINOPHYCEAE Fritsch en West \& Fritsch 1927

ORDEN: Dinophysiales Kofoid 1926

FAMILIA: Amphisoleniaceae Lindemann 1928

Amphisolenia bidentata Schröder 1900

Amphisolenia bifurcata Murray \& Whitting 1899

FAMILIA: Dinophysaceae Bütschli 1885

Dinophysis caudata Kent 1881

Ornithocercus magnificusStein 1883

Ornithocercus quadrates Schütt 1900
FAMILIA: Oxyphysiaceae Sournia 1984

Phalacroma doryphorum Stein 1883

Phalacroma favus Kofoid \& Michener 1911

Phalacroma mitra Schütt 1895

ORDEN: Gonyaulacales Taylor 1980

FAMILIA: Ceratiaceae Kofoid 1907

Tripos arietinus (Cleve) Gómez 2013

Tripos azoricus (Cleve) Gómez 2013

Tripos brevis (Ostenfeld \& Johannes Schmidt)

Gómez 2013

Tripos candelabrum (Ehrenberg) Gómez 2013

Tripos dens (Ostenfeld \& Johannes Schmidt)

Gómez 2013

Tripos furca (Ehrenberg) Gómez 2013

Tripos fusus (Ehrenberg) Gómez 2013

Tripos karstenii (Pavillard) Gómez 2013

Tripos lineatum (Ehrenberg) Gómez 2013

Tripos longinum (Karsten) Gómez 2013

Tripos longipes (Bailey) Gómez 2013

Tripos macroceros (Ehrenberg) Gómez 2013

Tripos massiliensis (Gourret) Gómez 2013

Tripos muelleri Bory en Lamouroux, Bory \&

Deslongschamps 1827

Tripos pentagonus (Gourret) Gómez 2013

Tripos reticulatus (Pouchet) Gómez 2013

Tripos vultur (Cleve) Gómez 2013

FAMILIA: Gonyaulacaceae Lindemann 1928 Gonyaulax Diesing 1866

FAMILIA: Ostreopsidaceae Lindemann 1928

Coolia monotis Meunier 1919

Ostreopsis lenticularis Fukuyo 1981

Ostreopsis siamensis Johs. Schmidt 1901

FAMILIA: Protoceratiaceae Lindemann 1928 Ceratocorys horrida Stein 1883

ORDEN: Gymnodiniales Apstein 1909

FAMILIA: Gymnodiniaceae Lankester 1885 Amphidinium longum Lohmann 1908 Amphidinium schroederi Schiller 1928

Gymnodinium sp. Stein 1878

Gymnodinium cinctum Kofoid \& Swezy 1921 
Gymnodinium gracile Bergh 1881

Gymnodinium lohmannii Paulsen 1908

Gyrodinium fusiforme Kofoid \& Swezy 1921

Levanderina fissa (Levander) Moestrup et al., 2014

ORDEN: Peridiniales Haeckel 1894

FAMILIA: Kolkwitziellaceae Lindemann, 1928

Diplopsalis lenticula Bergh 1881

FAMILIA: Heterodiniaceae Lindemann, 1928

Heterodinium Kofoid, 1906

FAMILIA: Heterocapsaceae Fensome et al., 1980 Heterocapsa triquetra (Ehrenberg) Stein 1883

FAMILIA: Protoperidiniaceae Balech 1988 Protoperidinium crassipes (Kofoid) Balech 1974 Protoperidinium depressum (Bailey) Balech 1974 Protoperidinium divergens (Ehrenberg) Balech 1974

Protoperidinium laticeps (Grontved \& Seidenfaden) Balech 1974

Protoperidinium oceanicum (Vanhöffen) Balech 1974

Protoperidinium ovatum Pouchet 1883

Protoperidinium pentagonum (Gran) Balech 1974

Protoperidinium rectum (Kofoid) Balech 1974

ORDEN: Prorocentrales Lemmermann 1910

FAMILIA: Prorocentraceae Stein 1883

Prorocentrum gracile Schütt 1895

Prorocentrum lima (Ehrenberg) Stein 1878

Prorocentrum micans Ehrenberg 1834

ORDEN: Pyrocystales Apstein 1909

FAMILIA: Pyrocystaceae (Schütt) Lemmermann 1899

Pyrocystis fusiformis Thomson en Murray 1876

Pyrocystis pseudonoctiluca Wyville-Thompson en Murray 1876

ORDEN: Thoracosphaerales Tangen 1982

FAMILIA: Thoracosphaeraceae Schiller 1930 Goniodoma sphaericum Murray \& Whitting 1899
Scrippsiella acuminate (Ehrenberg) Kretschmann et al., 2015

CLASE: NOCTILUCOPHYCEAE Fensome et al., 1993

ORDEN: Noctilucales Haeckel 1894

FAMILIA: Noctilucaceae Kent 1881

Noctiluca scintillans (Macartney) Kofoid \& Swezy 1921

FAMILIA: Protodiniferaceae Kofoid \& Swezy 1921

Pronoctiluca spinifera (Lohmann) Schiller 1932

\section{EUGLENOFITAS}

PHYLUM: EUGLENOZOA Cavalier-Smith 1981

CLASE: EUGLENOPHYCEAE Schoenichen en Eyferth \& Schoenichen 1925

ORDEN: Euglenales Bütschli 1884

FAMILIA: Euglenaceae Dujardin 1841

Euglena sp. Ehrenberg 1830

FAMILIA: Phacaceae Kim, Triemer \& Shin 2010

Phacussp. Dujardin 1841

ORDEN: Eutreptiales Leedale 1967

FAMILIA: Eutreptiaceae Hollande 1942

Eutreptiella gymnastica Throndsen 1969

PHYLUM: OCHROPHYTA Cavalier-Smith en Cavalier-Smiith \& Chao 1996

CLASE: DICTYOCHOPHYCEAE Silva 1980

ORDEN: Dictyochales Haeckel 1894

FAMILIA: Dictyochaceae Lemmermann 1901

Dictyocha fibula Ehrenberg 1839

CLASE: RAPHIDOPHYCEAEChadefaud ex Silva 1980

ORDEN: Chattonellales Throndsen 1993

FAMILIA: Chattonellaceae Throndsen 1993

Chattonella marina (Subrahmanyan) Hara \& Chihara 1982 
FAMILIA: Fibrocapsaceae Cavalier-Smith en Cavalier-Smith \& Scoble 2013

Fibrocapsa japonica Toriumi \& Takano 1973

\section{CIANOBACTERIAS}

PHYLUM: CYANOBACTERIA Stanier ex Cavalier-Smith 2002

CLASE: CYANOPHYCAEA Schaffner 1909

ORDEN: Nostocales Borzì 1914

FAMILIA: Nostocaceae Eichler 1886 Anabaena Bory ex Bornet \& Flahault 1886

FAMILIA: Rivulariaceae Bornet \& Flahault 1886 Rivularia sp. Agardh ex Bornet \& Flahault 1886

ORDEN: Oscillatoriales Schaffner 1922

FAMILIA: Microcoleaceae Strunecky et al., 2013 Trichodesmium thiebautii Gomont ex Gomont 1890

FAMILIA: Oscillatoriaceae Engler 1898 Lyngbya sp. Agardh ex Gomont 1892 Oscillatoria sp. Vaucher ex Gomont 1892

ORDEN: Spirulinales Komárek et al., 2014 FAMILIA: Spirulinaceae (Gomont) Hoffmann, Komárek \& Ka en Komárek et al., 2014 Spirulina sp. Turpin ex Gomont 1892

\section{Discusión de resultados}

En esta zona del país, no se han realizado estudios sobre la estructura comunitaria del fitoplancton marino, por lo que esta investigación representa un aporte para el conocimiento de su biodiversidad, como parte de un estudio de línea base ambiental. Las microalgas planctónicas constituyen la base de la red trófica, sobre la cual, prosperan los demás elementos que la conforman, debido a que representan la puerta de entrada de la energía solar en el ecosistema pelágico y la base de su mantenimiento. Son fotosintéticos y los principales productores de biomasa y compuestos orgánicos en los océanos (Livingston, 2001).
En cuanto a la estructura comunitaria, la zona en estudio muestra gran heterogeneidad, debido a la presencia de especies oceánicas, tales como la dinofícea: Ornithocercus y bentónicas, como: Diplopsalis, Ostreopisis, Pleurosigma y Odontella, lo cual indica la ocurrencia de procesos de mezcla en la zona (Vila, Garces \& Masó, 2001). Por otra parte, la presencia de las cianofitas: Trichodesmium thiebauthii, Lyngbya birgei, Oscillatoria, Rivularia y Spirulina, así comolas euglenofitas: Euglena, Eutreptiella y Phacus, las cuales, son indicadoras de grandes aportes de materia orgánica; evidencia una influencia de aguas de origen doméstico provenientes de las operaciones de la base militar que ahí se encuentra, sin embargo, la dinámica de las corrientes permitiría que estas aguas sean muy diluidas generando muy poco impacto ambiental.

Al comparar con otros estudios realizados en las islas oceánicas del mar Caribe, se consiguen riquezas similares, por ejemplo Pérez-Castresana, Villamizar, Varela y Fuentes, (2014) en su estudio realizado en el Parque Nacional Archipiélago Los Roques, ubicado en la zona central del mar Caribe Venezolano, identificaron 67 especies de 211 morfotipos observados, incluyendo diatomeas de los siguientes géneros: Asterionella, Campylodiscus, Dictyoneis, Ditylum, Guinardia, Haslea, Isthmia, Leptocylindrus, Pseudo-nitzschia, Skeletonema, Striatella, Thalassiosira, Trapidoneis y Triceratium; los dinoflagelados: Ceratocoris, Diplopsalis, Gonyodoma y Scripsiella y las cianobacterias; Lyngbya y Trichodesmium.

Margalef (1969) en su estudio del ecosistema pelágico del NE de Venezuela registró 300 especies fitoplanctónicas, y menciona que con el aporte de otros autores y la información publicada de otras zonas del Caribe, no menos de 450 especies podrían ser encontradas. Actualmente existen trabajos sobre los principales grupos de fitoplancton que citan un mayor número de taxa para el mar Caribe. Para el área del Caribe colombiano se han registrado 523 taxón, identificados en varios estudios 
a lo largo de la costa, de los cuales 337 taxón corresponden a diatomeas y 186 taxa a dinoflagelados (Lozano-Duque et al., 2010).

Navarro y Hernández-Becerril (1997), con base en una revisión de literatura disponible para el área total del mar Caribe, encontraron 1,083 taxa de diatomeas marinas, 305 taxa pertenecientes a diatomeas centrales y 778 taxa pertenecientes a diatomeas pennales. El número de especies de microalgas planctónicas en el presente estudio es muy bajo en comparación a los citados anteriormente, sin embargo, hay que considerar que Isla de Aves corresponde a una isla coralina alejada de la plataforma continental y que el resto de los estudios incluye zonas productivas por la ocurrencia de surgencia costera y zonas costeras que incluyen ciénagas, lagunas y descargas de ríos al mar.

En general, el fitoplancton estuvo representado por las diatomeas, tanto en número de especies como en abundancia, que en general, suelen ser el grupo más abundante de la comunidad fitoplanctónica, especialmente en áreas costeras, debido a su capacidad de asimilación rápida de nutrientes y su alta tasa reproductiva, lo cual les da el calificativo de especies oportunistas (Triantafyllou et al., 2001; Cetin \& Sen, 2004).

Entre las diatomeas más relevantes en el estudio, se encontraron Asteroplanus karianus, Coscinodiscus perforatus, Skeletonema costatum, Leptocylindrus minimus, Nitzschia sp., Leptocylindrus danicus, Asterionella gracilis, Chaetoceros dydimus y Haslea waiwrikae, las cuales, son especies costeras indicadoras de aguas turbulentas (Margalef, 1965). En cuanto a la diatomea Skeletonema costatum, su presencia en la zona este del mar Caribe indica la influencia de aguas del río Amazonas, por lo que $S$. costatum se asocia a aguas salobres por el aporte de grandes ríos (Ferguson-Wood, 1968). Además de las especies netamente planctónicas, se detectaron especies bentónicas, las cuales sugieren la ocurrencia de fenómenos de mezcla de la columna de agua, que ejercen un intercambio fuerte de especies bentónicas y pelágicas (Lucas, Vidal \& Navas, 2001).

En este mismo orden de ideas, Spiniello (1996) señala que el grupo Chrysophyceas a nivel nacional, presenta una riqueza de 385 especies y 25 variedades agrupadas en 99 géneros, de los cuales Coscinodiscus, Chaetoceros, Navicula sp. y Nitzschia $s p$., reúnen a más del $35 \%$ de las especies, tal como se observa en los resultados obtenidos en este estudio. Por su parte, el género Nitzschia crece en ambientes ricos en nutrientes y son capaces de modificar estas condiciones para hacerlo apropiado para el desarrollo de otros representantes del fitoplancton (Barber \& Ryther, 1969).

Asimismo, los géneros: Chaetoceros, Leptocylindrus, Nitzschia y Rhizosolenia, son oportunistas y por lo tanto, indicadoras de las primeras etapas de una sucesión ecológica, y el género Asterionellopsis, acompañado de altas densidades de dinoflagelados y cianofitas puede indicar un ambiente con altas concentraciones de nutrientes, pero sin condiciones de eutrofización, característico de aguas afloradas (Margalef, 1969; Bellinger \& Sigee, 2010).

Los dinoflagelados constituyen el segundo grupo más representativo en cuanto a riqueza. Estos, representan solo un porcentaje del total del número de organismos donde dominan las diatomeas, a pesar de ser organismos ventajosos respecto a estas, ya que presentan una movilidad que le permite realizar desplazamientos para la búsqueda de nutrientes y áreas con intensidad de luz óptima (Lindenschmidt \& Chorus, 1998).

En cuanto a la presencia de la cianobacteria Trichodesmium thiebauthii, este grupo ha sido asociado a fenómenos de eutrofización, debido a su capacidad de sobrevivir en cuerpos de agua con altos niveles de materia orgánica. Aunque esta especie suele ser característica de regiones oligotróficas y un productor primario importante en el océano Atlántico tropical (Staal, Meysman, \& Stal, 2003). En general, las cianobacterias han sido usadas como 
indicadoras de calidad de agua, específicamente de entradas de nutrientes, eutrofización y diferentes formas de contaminación (Paerl \& Tuker, 1995). A pesar que la zona presenta poca intervención humana, se considera muy productiva debido a los productos fecales de las aves que ahí habitan, los cuales contribuyen a un incremento en las concentraciones de nutrientes como el fósforo.

En cuanto a la abundancia fitoplanctónica, se obtuvo una densidad más de 40 veces por debajo de los 65,000 células/litro, establecidos para catalogar aguas oligotróficas. De hecho, las densidades estimadas en este estudio están 10 veces por debajo de la obtenida por Pérez-Castresana et al. (2014), en el Parque Nacional Archipiélago Los Roques, y en el mismo orden de magnitud, estimado en la zona de Palanquines en la Isla La Tortuga (RodríguezCenteno, Díaz-Ramos, Charzeddine, Subero-Pino \& Troccoli-Ghinaglia, 2010), lo cual establece un indicador local para las islas oceánicas del mar Caribe venezolano, que se caracterizan por presentar aguas oligotróficas, propias de ecosistemas coralinos.

Asimismo, Marshal y Solder (1982) indicaron que a partir de los estudios hechos por el crucero Oregón en el mar Caribe, se obtuvo densidades medias del fitoplancton en las estaciones del este de 13,382 células/litro, 2,015 cél/l en la zona este central, 1,205 cél/l en la zona oeste central y 1,026 cél/l en las estaciones ubicadas al oeste, lo cual coincide con lo encontrado en este estudio.

En general, la zona marina y costera de Isla de Aves se caracteriza por una comunidad fitoplanctónica diversa, característica de aguas turbulentas, pero para entender mejor estos procesos es necesario el estudio de las condiciones fisicoquímicas del medio y su interrelación con la comunidad planctónica, por lo que se recomienda hacer este tipo de estudios en otras épocas del año, en la que ocurren procesos meteorológicos distintos, tales como tormentas y huracanes, los cuales cambian las condiciones fisicoquímicas del agua y por ende de sus comunidades biológicas.

\section{Agradecimientos}

Los autores ofrecen un agradecimiento especial al personal de la Base Científico Naval Simón Bolívar, por toda la colaboración brindada durante la ejecución de las campañas científicas, así como al Servicio de Hidrografía y Navegación (SHN), la Cancillería de la República Bolivariana de Venezuela y el Ministerio del Poder Popular para el Ecosocialismo; por la organización de las campañas científicas y el apoyo logístico para la participación de las universidades e instituciones de investigación del país.

\section{Referencias bibliográficas}

Al-Yamani, F. \& Saburova, M. (2011). Illustrated guide on the benthic diatoms of Kuwait's Marine Environment. Kuwait: Kuwait Institute for Scientific Research.

Arrigo, K., Robinson, D., Worthen, D., Dunbar, R., Ditullio, G., Vanwoert, M. \& Lizotte, M. (1999). Phytoplankton community structure and the drawndown of nutrients and $\mathrm{CO} 2$ in the Southern Ocean. Science, 283: 365 - 367. doi: 10.1126/science.283.5400.365.

Balech, E. (1988). Los dinoflagelados del Atlántico Sudoccidental. Publicaciones del Instituto Español de Oceanografi. Madrid: Ministerio de Agricultura, Pesca y Alimentación. Secretaría General Técnica.

Barber, R. \& Ryther, J. (1969). Organic chelators: factors affecting primary production in the cromwell current upwelling. Journal of Experimental Marine Biology and Ecology 3: 191 - 199. doi: 10.1016/0022-0981(69)90017-3.

Bellinger, E. \& Sigee, D. (2010). Freshwater algae. Oxford: Walley-Blackwell. 
Berárd-Therriault, L., Poulin, M. \& Bossé, L. (1999). Guide d' identification du phytoplankton marin de lèstuarie et du Golfe de Saint-Laurent. Incluant également certains protozoaries. Otawa: Conseil Nacional de Recherches du Canadá.

Cetin, M. \& Sen, M. (2004). Seasonal distribution of phytoplankton in Orduzu Dam Lake (Malatya, Turkey). Turk Journal Botanical 28: 279-285. doi: 10.1.1.685.9868.

Chretiennot-Dinet, M., Sournia, A., Ricard, M. \& Billard, C. (1993). A classification of the marine phytoplankton of the world from class to genus. Phycologa 32 (3): 159-179. doi: 10.2216/ i0031-8884-32-3-159.1.

Cupp, E. (1943). Marine plankton diatoms of the West Coast of North America. Bulletin of the Scripps Institution of Oceanography of the University of California. 5(1), 1-238. Recuperado de http://aquaparadox.obs-vlfr.fr/html/PFD/ Taxonomic\%20Monographs/Cupp1973.pdf

Ferguson, J. (1968). Dinoflagellates of the Caribbean Sea and adjacent areas. University of Miami Press. Florida.

Ferguson-Wood, E. (1968). Studies of phytoplankton ecology in tropical and subtropical environments of the Atlantic Ocean. Part 3. Phytoplankton communities in the Providence channels and the Tongue of the Ocean. Bulletin of Marine Science 18 (2): 481-543.

Gruber, W. (2003). Isla de Aves. (Trabajo de Ascenso). Universidad Central de Venezuela, Venezuela.

Guada, H. \& Solé, G. (2000). Plan de Acción para la Recuperación de las Tortugas Marinas de Venezuela. Suárez, A. (ed.). (Informe Técnico del PAC No. 39). UNEP, Caribean EnvirometProgramme, Kingston, Jamaica.

Guiry, M. \& Guiry, G. (2018). AlgaeBase. Worldwide electronic publication, National University of
Ireland, Galway. Recuperado de http://www.algaebase.org.

Hammer, O., Harper, D. \& Ryan, P. (2001). PAST: Paleontological Statistics software package for education and data analysis. Paleantología Electrónica, 4 (1), 9. doi: 10.1.1.459.1289.

Hubschmannat et al., (1988). Isla de Aves, Bastión venezolano en el mar Caribe. Caracas: Dirección de Geografía y Cartografía Militar, Ministerio de la Defensa.

Lindenschmidt, K. \& Chorus, I. (1998). The effects of water column mixing on phytoplankton succession, diversity and similarity. Journal of Plankton Research 20(10): 1927-1951. doi: 10.1093/plankt/20.10.1927.

Livingston, R. (2001). Eutrophication processes in coastal systems: origin and succession of plankton blooms and effects on secondary production in Gulf Coast Estuaries. Center for Aquatic Research and Resource Management. Press. Florida: Florida State University, CRC Press.

Lozano-Duque, Y., Vidal, L. \& Navas, G. (2010). La comunidad fitoplanctónica en el mar Caribe colombiano. En: INVEMAR (eds.). 2010. Biodiversidad del margen continental del Caribe colombiano. Santa María: Serie de Publicaciones Especiales, Invemar.

Lucas, C., Banham, C. \& Holligan, P. (2001). Benthic-pelagic exchange of microalgae at a tidal flat. 2. Taxonomic analysis. Marine Ecology 212: 39-52. doi: 10.3354/meps212039.

Luz, B. \& Barkan, E. (2000). Assessment of Oceanic productivity with the triple-isotope composition of dissolved oxygen. Science 288: 20282031. doi: 10.1126/science.288.5473.2028.

Margalef, R. (1965). Composición y distribución del fitoplancton.Sociedad de Ciencias Naturales La Salle 25 (70-72): 141 - 205. 
Margalef, R. (1969). El ecosistema pelágico del mar Caribe. Sociedad de Ciencias Naturales La Salle 29(82): 5 - 36.

Marshall, H. \& Solder, J. (1982). Pelagic phytoplankton of the Caribbean Sea. Bulletin of Marine Science 32: 354 - 365.

Navarro, J. \& Hernández-Becerril, D. (1997). Listados Floristicos de México. XV. Check-list of Marine Diatoms from the Caribbean Sea. Ciudad de México: UNAM, Instituto de Biología.

Paerl, H. \& Tucker, C. (1995). Ecology of bluegreen algae in acuacultura ponds. Journal of the World Aquaculture Society 26(2): 109-131. doi: 10.1111/j.1749-7345.1995.tb00235.x.

Peragallo, M. (1965). Diatomés marines du France. Amsterdam: A Asher Co.

Pérez-Castresana, G., Villamizar, E., Varela, R. \& Fuentes, Y. (2014). Descripción preliminar del fitoplancton en seis arrecifes coralinos del parque nacional Archipiélago de Los Roques. Acta Biológica Venezuelica 34(2): 293-309.

Rodríguez-Centeno, M., Díaz-Ramos, J., Charzeddine, L., Subero-Pino, S. \& TroccoliGhinaglia, L. (2010). Abundancia del fitoplancton en la isla la Tortuga, Dependencia Federal, Venezuela. Boletin do Instituto Oceanográfico 49 (1): 77-86.

Round, F., Crawford, R. \& Mann, D. (1990). The Diatoms, Biology \& Morphology of the Genera. Cambridge: Cambridge University Press.

Saunders, R. \& Glenn, E. (1969). Diatoms. Memoirs of the Hourglass Cruises. VolX. Florida: Department of Natural Resources Marine Research Laboratory. St. Petersburg.

Siqueiros, D., Valenzuela, A., Hernández, O., Argumedo, U. \& López, F. (2004). Catálogo iconográfico de diatomeas de hábitats rocosos y su incidencia en la dieta de abulones (Haliotis spp.) jóvenes de Baja California Sur, Oceánides 19 (2): 29-103.

Siqueiros, D. \& Hernández, O. (2006). Floristics of epiphytic diatoms in a patch of subtropical macroalgae.CICIRMAROceánides 21(1,2): 11-61.

Soler, A., Pérez, M. \& Aguilar, E. (2003). Diatomeas de las costas del pacífico en Panamá. Agenda del Centenario. Universidad de Panamá-Vicerectoría de Investigación y Postgrado. Panamá

Sournia, A. (1986). Atlas du phytoplankton marin. Vol 1.París: Centre National de la Recherche Scientifique.

Spiniello, P. (1996). Las diatomeas marino-costeras de Venezuela. Caribbean Journal of Science 32 (4): 331-347.

Staal, M., Meysman, F. \& Stal, L. 2003. Temperature excludes $\mathrm{N}_{2}$-fixing heterocystous cyanobacteria in the tropical oceans. Nature 425: 504507. doi: 10.1038/nature01999.

Tomas, C. (1996). Identifying marine diatoms and dinoflagellates. New York: Academic Press.

Triantafyllou, G., Petihakis, G., Dounas, C. \& Athanasios, T. (2001). Assessing marine ecosystem response to nutrient imputs. Marine Pollution Bulletin 43(7-12): 175-186. doi: 10.1016/ S0025-326X(01)00071-6.

Troccoli, L. (2001). Cambios estructurales del fitoplancton costero tropical en una zona cárstica: Perspectivas en escala espacial. (Tesis de doctorado). Centro Nacional y de Estudios Avanzados del Instituto Politécnico Nacional Unidad Mérida. Yucatán, México.

Vila, M., Garces, E. \& Masó, M. (2001). Potentially toxic epiphytic dinoflagellate assemblages on macroalgae in the NW Mediterranean. Aquatic Microbial Ecology 26: 51-60. doi: 10.3354/ ame026051. 
Yamaji, I. (1996).Illustration of the marine plankton of Japan. Osaka: Hoikusha Publishers.

Yilmaz, N., Yardimci, C., Elhag, M. \& Dumitrache, C. (2018). Phytoplankton Composition and Water Quality of Kamil $\mathrm{Abdu}_{s} \mathrm{~s}$ Lagoon (Tuzla Lake), Istanbul-Turkey. Water 10(5), 603doi: 10.3390/ w10050603.
Yranzo, A., Villamizar, E., Romero, M. \& Boadas, H. (2014). Estructura de las comunidades de corales y octocorales de Isla de Aves, Venezuela, Caribe Nororiental. Revista de Biología Tropical 62(3): 115-136. doi: 10.15517/rbt.v62i0.15907. 